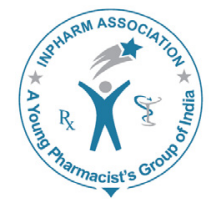

\title{
Chronic administration of ketamine induces depression in Wistar rat, but does not affect sexual function
}

Sir,

Schizophrenia is a mental disorder that is characterized by periodic shift in positive symptoms (conceptual disorganization, delusions, hallucinations, hyperactivity, stereotypy behaviour etc.) and negative symptoms (social withdrawal, loss of function, anhedonia, decreased emotional expression, impaired concentration, and diminished social engagement). ${ }^{1,2}$ Anhedonia in experimental rats is marked by decrease in intracranial self-stimulation, consumption of sucrose solution, and sexual behavior. ${ }^{3}$ Depression is associated with different degrees of sexual dysfunction and population suffering from sexual dysfunction are frequently depressed. ${ }^{3,4}$ An acute dose of ketamine, a noncompetitive NMDA receptor antagonist is reported to relieve pain induced depression while a chronic dose of ketamine is reported to induce depression. ${ }^{5,1,2}$ Administration of single dose of dizocilpine maleate (MK-801), an NMDA antagonist was reported to impair sexual function in sexually naive and sexually experienced male rats ${ }^{6}$, but the effect of chronic administration of ketamine on depression induced sexual function was not studied. Therefore, the present study was designed to test the effect of chronic administration of ketamine on depression and sexual function, using forced swim test and sexual behavior study respectively. ${ }^{1,2,7}$ While Powell et al. studied effect of single dose of NMDA antagonist on sexual function; we studied effect of chronic ketamine (another NMDA antagonist) dosing on depression and effect of depression on sexual function.

Rats were maintained as per standard laboratory condition and experimental protocol was approved by animal ethics committee of the institute. Before dosing, male rats were provided training three times in a week by placing them with estrous female rats (1:1) for 30 minutes. The male rats failing to mount and intromit (vaginal penetration),

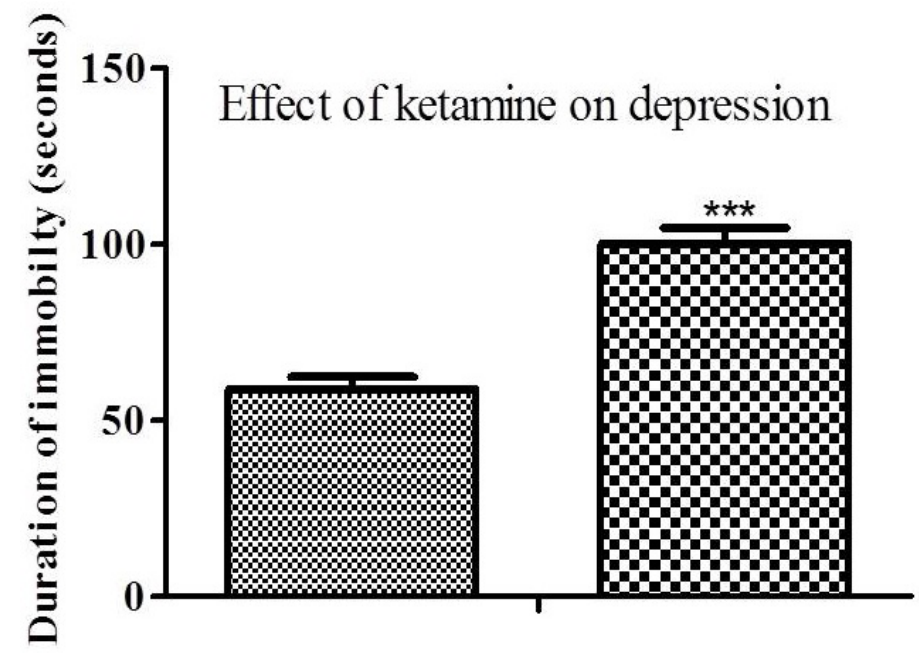

Rat treated with water $\mathbf{0}$ Rat treated with ketamine

Figure 1: Duration of immobility in rats treated with vehicle and ketamine.

Values are expressed as mean \pm SEM, $n=6,{ }^{* * *} p<0.001$. 
Table 1: Sexual behavior in rats treated with vehicle and ketamine.

\begin{tabular}{lll} 
Sexual behavior & Rats treated with water & $\begin{array}{l}\text { Rats treated with } \\
\text { ketamine }\end{array}$ \\
\hline ML (seconds) & $56.1 \pm 4.7$ & $57.1 \pm 7.2$ \\
\hline IL (seconds) & $93.1 \pm 6.1$ & $89.8 \pm 4.1$ \\
\hline MF (number) & $7.8 \pm 0.6$ & $7 \pm 0.4$ \\
\hline IF (number) & $4 \pm 0.4$ & $3.6 \pm 0.6$ \\
\hline EL (seconds) & $455.8 \pm 24.9$ & $476.6 \pm 35.9$ \\
PEI (seconds) & $328.8 \pm 16.2$ & $321.6 \pm 19.3$
\end{tabular}

Values are expressed as mean \pm standard error of mean, $n=6$.

and female rats not showing lordosis posture (a sign of sexual excitement) were excluded from the study and were replaced by equal number of same sex animals. Six male rats were given $30 \mathrm{mg} / \mathrm{kg}$ of Ketamine hydrochloride (Neon Laboratories Limited, India) intraperitonally at $4.30 \mathrm{pm}$ every day for 10 days where as another 6 male rats received distilled water intraperitoneally for the same duration. On $9^{\text {th }}$ day of dosing male animals were forced to swim for 15 minutes at $5 \mathrm{pm}$ and on $10^{\text {th }}$ day same animals were forced to swim for 5 minutes at the same time and total time of immobility was noted. Animals were dried using towels and room heater. Thirty minutes after forced swim test, male animals were subjected to sexual behavior study in presence of estrous female rats. Mount latency/ML (time taken by male rats to assume copulatory position on female rat), intromission latency/IL (time taken by male rats to copulate

\section{REFERENCES}

1. Chatterjee M, Ganguly S, Srivastava M, Palit G. Effect of 'chronic' versus 'acute' ketamine administration and its 'withdrawal' effect on behavioural alterations in mice: Implications for experimental psychosis. Behav Brain Res. 2011; 216(1): 247-54.

2. Chatterjee M, Jaiswal M, Palit G. Comparative evaluation of forced swim test and tail suspension test as models of negative symptom of schizophrenia in rodents. ISRN Psychiatry. 2012; 2012: Article ID 595141.

3. Bonilla-Jaime H, Retana-Marquez S, Velazquez-Moctezuma J. Pharmacological features of masculine sexual behavior in an animal model of depression. Pharmacol Biochem Behav. 1998; 60(1): 39-45.

4. Seidman SN, Roose SP, Menza MA, Shabsigh R, Rosen $\mathrm{RC}$. Treatment of erectile dysfunction in men with depressive with female rat), mount frequency/MF (number of mount in a sexual cycle), intromission frequency/IF (number of intromission in a given sexual cycle), ejaculation latency (time taken from the first intromission till ejaculation), post-ejaculatory interval/PEI (time taken by male rat from first ejaculation till next intromission).

For statistical calculation, values are expressed as mean \pm standard error of mean (SEM) and students t-test was employed for calculating statistical significance where $\mathrm{p}<$ 0.05 was considered as statistically significant.

Rats developed depression after ten days administration of ketamine as evident by significant $(\mathrm{p}<0.001)$ increase in immobility time in forced swim test in comparison to control rats (Figure 1), but there was no statistically significant change in sexual behavior of these rats (Table 1). Presently, we cannot explain as to why ketamine does not induce sexual dysfunction in Wistar rats where as an NMDA receptor antagonist (MK-801) impairs sexual function of the Long-Evans/Blue Spruce rats. This could be due to change in species of rats. A detailed and long term study is required to know the effect of ketamine on sex hormone (like testosterone), enzymes (phosphodiesterase type 5, Rho-kinase 2 etc.), neurotransmitters (nitric oxide and dopamine) and ion channels (potassium and calcium) and its role in sexual function. ${ }^{6,8}$ symptoms: results of a placebo-controlled trial with sildenafil citrate. Am J Psychiatry. 2001; 158(10): 1623-30.

5. Wang J, Goffer Y, Xu D, Tukey DS, Shamir DB, Eberle SE, et al. A single sub-anesthetic dose of ketamine relieves depression like behaviors induced by neuropathic pain in rats. Anesthesiology 2011; 115(4): 812-21.

6. Powell WS, Dominguez JM, Hull EM. An NMDA antagonist impairs copulation and the experience-induced enhancement of male sexual behavior in the rat. Behav Neurosci. 2003; 117(1): 69-75.

7. Agmo A. Male rat sexual behavior. Brain Res Brain Res Protoc. 1997; 1(2): 203-9.

8. Anderson KE. Pharmacology of penile erection. Pharmacol Rev. 2001; 53(3): 417-50.

\section{Sumanta Kumar Goswami, Archana Kossireddi Venkata, Noor Zahra Jaffer, Mohammed Naseeruddin Inamdar.}

Department of Pharmacology, Al-Ameen College of Pharmacy, Bangalore, India - 560027.

DOI : 10.5530/jyp.2015.2.13 